\title{
High GDF-8 in follicular fluid is associated with a low pregnancy rate in IVF patients with PCOS
}

\author{
Lanlan Fang*, Sijia Wang*, Yiran Li, Yiping Yu, Yuxi Li, Yang Yan, Jung-Chien Cheng and \\ Ying-Pu Sun \\ Center for Reproductive Medicine, Henan Key Laboratory of Reproduction and Genetics, The First Affiliated \\ Hospital of Zhengzhou University, Zhengzhou, China
}

Correspondence should be addressed to L Fang or Y-P Sun; Email: fanglly@163.com or syp2008@vip.sina.com

$*(\mathrm{~L}$ Fang and S Wang contributed equally to this work)

\begin{abstract}
Polycystic ovary syndrome (PCOS) is the most common cause of female infertility. Growth differentiation factor-8 (GDF-8) is expressed in the ovary and can be detected in human follicular fluid which provides an important microenvironment for maintaining physiological functions of the ovarian follicle. To date, the relationship between GDF-8 levels in follicular fluid and the risk of PCOS is completely unknown. In the present study, we show that during the process of the controlled ovarian hyperstimulation (COH), serum GDF-8 levels are higher on the day of gonadotropin administration and 14 days after embryo transfer in in vitro fertilization (IVF) patients with PCOS than they are in IVF patients without PCOS. Importantly, GDF-8 levels in follicular fluid at oocyte retrieval are also higher in PCOS patients than in non-PCOS patients. Treatment of primary human granulosa-lutein (hGL) cells with GDF-8 downregulates StAR protein expression and the inhibition is more pronounced in hGL cells from PCOS patients than it is in cells from non-PCOS patients. Importantly, high GDF-8 levels and low progesterone (P4) levels were associated with poor pregnancy outcomes in PCOS patients. Our results provide the first evidence that aberrant expression of GDF-8 in the follicular fluid of PCOS patients results in abnormal P4 expression, which leads to poor pregnancy outcomes.
\end{abstract}

Reproduction (2020) $\mathbf{1 6 0} 11-19$

\section{Introduction}

Reproductive disorders have significant medical, social and economic impacts on individuals and society. Polycystic ovary syndrome (PCOS) is a hormonal disorder that affects reproductive age women, and it is the most common cause of infertility, affecting $5-10 \%$ of women. Clinically, PCOS is characterized by polycystic ovaries verified by ultrasonography, hyperandrogenism and ovulatory dysfunction (McCartney \& Marshall 2016). To date, the exact cause of PCOS remains unclear. However, several genetic, hormonal and environmental factors have been reported to be involved in the pathogenesis of PCOS (Dumesic et al. 2015, Witchel et al. 2019). Emerging evidence demonstrates that many extra- and intra-ovarian factors can affect folliculogenesis, oocyte maturation, quality of embryo and pregnancy outcomes, and dysregulations of these factors subsequently contribute to the development of PCOS (Qiao \& Feng 2011).

Endocrine factors such as FSH and LH play pivotal roles in regulating female reproductive functions. However, normal ovarian function also depends on a number of locally produced factors that exert their effects in an autocrine and/or a paracrine fashion. The transforming growth factor-beta (TGF- $\beta$ ) superfamily consists of TGF- $\beta \mathrm{s}$, growth and differentiation factors (GDFs), bone morphogenetic proteins (BMPs), activins, inhibins, anti-Mullerian hormone (AMH) and other proteins. Many members of the TGF- $\beta$ superfamily are secreted by ovarian cells, where they can regulate various physiological and pathological events in the ovary (Knight \& Glister 2006). Aberrant expression of activin $A$, inhibin $A / B, A M H, G D F-9$ and BMP-15 has been observed in the serum or follicular fluid of PCOS patients and can contribute to the pathogenesis of PCOS (Qiao \& Feng 2011).

Growth differentiation factor-8 (GDF-8) is a member of the TGF- $\beta$ superfamily; GDF-8 is also known as myostatin, and it was first identified in muscle tissue and reported as a negative regulator of muscle cell growth and differentiation (McPherron \& Lee 1997, McPherron et al. 1997). We and others have demonstrated that GDF-8 and its putative receptors (ALK5, ACVR2A and ACVR2B) are expressed in human granulosa cells and that the protein levels of GDF-8 can be detected in serum and follicular fluid (Chang et al. 2015, Fang et al. 2016, Lin et al. 2018). In addition, GDF-8 is able 
to regulate cumulus expansion, steroidogenesis, oocyte maturation, and granulosa cell proliferation (Chang et al. 2015, 2016a,b,c, Fang et al. 2015). Our previous study showed that serum GDF-8 levels are dynamically changed in patients undergoing controlled ovarian hyperstimulation $(\mathrm{COH})$ during in vitro fertilization (IVF) treatment. In addition, the levels of serum GDF-8 play a critical role in ensuring successful pregnancy because they maintain appropriate serum progesterone (P4) levels (Fang et al. 2016).

To the best of our knowledge, there have been only three studies that examined the expression levels of GDF-8 in serum of women with PCOS. Serum GDF-8 levels are higher in women with PCOS than in those without PCOS (Chen et al. 2012). In addition, an immunohistochemical study demonstrated that the expression levels of GDF-8 and its functional receptors are higher in antral follicles of PCOS patients than those of non-PCOS patients (Lin et al. 2018). In contrast, a recent study showed that serum GDF-8 levels are slightly lower in PCOS patients than in non-PCOS patients, although no significant differences were detected (Arpaci 2019). Follicular fluid provides an important microenvironment for maintaining physiological functions of the ovarian follicle. It is known that GDF-8 is expressed in the follicular fluid. However, the relationship between GDF-8 levels in follicular fluid and the risk of PCOS remains unknown. Given the impacts of GDF-8 levels and PCOS on pregnancy outcomes, the present study was designed to investigate the changes in GDF-8 levels in the human follicular fluid of IVF patients with PCOS and examine the effect of that on the pregnancy outcomes.

\section{Materials and methods Antibodies and reagents}

Polyclonal anti-StAR antibody was obtained from Santa Cruz Biotechnology. Monoclonal mouse anti- $\alpha$-tubulin antibody was obtained from CMCTAG (Shanghai, China). Horseradish peroxidase-conjugated goat anti-rabbit and goat anti-mouse IgGs were obtained from Abcam. Recombinant human GDF-8 was obtained from R\&D Systems.

\section{Research ethical statement}

The study received institutional approval (reference number: 2019-KY-48) and was carried out in accordance with the guidelines from the Zhengzhou University Research Ethics Board. Written informed consent was obtained from all of the subjects before participation in the study.

\section{Human serum and follicular fluid samples}

Human serum and follicular fluid samples were obtained from 80 patients (40 non-PCOS and 40 PCOS patients) who received IVF treatment with fresh embryo transfer. The diagnosis of PCOS was based on the revised Rotterdam diagnostic criteria for PCOS (Rotterdam 2004). Clinical and hormonal data from all included patients are shown in the Table 1. Causes of infertility were tubal obstruction or male infertility in both non-PCOS and PCOS patients. Patients with endometriosis, diminished ovarian reserve, chromosome abnormality, or hydrosalpinx were excluded from the study. All patients were treated with a standard long protocol. At the mid-luteal phase ( 7 days before next menstrual cycle), the gonadotropinreleasing hormone $(\mathrm{GnRH})$ agonist triptorelin $(0.1 \mathrm{mg})$ (Ipsen Pharma Biotech, France), was administered subcutaneously daily. Approximately 14 days after $\mathrm{GnRH}$ agonist injection was started, recombinant FSH (Gonal-F; Merck) was administered daily at a dosage of 150-300 IU. When at least three follicles had reached 18 mm, hCG (10,000 IU, Livzon, Zhuhai, China) was injected. Oocyte retrieval was scheduled approximately 34-36 $\mathrm{h}$ after hCG injection by transvaginal ultrasound-guided follicular aspiration. The follicular fluid was collected when the oocytes were retrieved. Only the first follicular fluid aspirate without blood or flushing solution was used for analysis. After $10 \mathrm{~min}$ of centrifugation at $270 \mathrm{~g}$, the supernatant was stored at $-80^{\circ} \mathrm{C}$ until further analysis. Blood samples were obtained by venipuncture at seven time points during $\mathrm{COH}$

Table 1 Clinical and hormonal data from non-PCOS and PCOS patients.

\begin{tabular}{lccc}
\hline & Non-PCOS $(n=40)$ & PCOS $(n=40)$ & $P$ \\
Age & $29.25 \pm 0.58$ & $29.28 \pm 0.55$ & 0.975 \\
BMI & $22.19 \pm 0.52$ & $24.43 \pm 0.52$ & 0.0034 \\
AFC & $16.65 \pm 0.87$ & $22.9 \pm 0.59$ & $<0.0001$ \\
Basal serum FSH $(\mathrm{mlU} / \mathrm{mL})$ & $6.56 \pm 0.26$ & $6.67 \pm 0.35$ & 0.794 \\
Basal serum LH $(\mathrm{mlU} / \mathrm{mL})$ & $5.34 \pm 0.32$ & $11.47 \pm 1.03$ & $<0.0001$ \\
Basal serum E2 $(\mathrm{pg} / \mathrm{mL})$ & $42.57 \pm 6.15$ & $40.26 \pm 3.50$ & 0.7454 \\
Basal serum P4 $(\mathrm{ng} / \mathrm{mL})$ & $0.51 \pm 0.07$ & $0.57 \pm 0.05$ & 0.549 \\
Basal serum T $(\mathrm{ng} / \mathrm{mL})$ & $0.25 \pm 0.02$ & $0.44 \pm 0.03$ & $<0.0001$ \\
Basal serum PRL $(\mathrm{ng} / \mathrm{mL})$ & $18.53 \pm 1.57$ & $17.36 \pm 1.05$ & 0.541 \\
Total Gn $($ recombinant FSH) dose $(\mathrm{IU})$ & $2083 \pm 139.1$ & $1751 \pm 112.5$ & 0.066 \\
The number of oocytes retrieved & $15.05 \pm 0.99$ & $13.6 \pm 0.90$ & 0.283 \\
The number of MIl oocytes & $12.48 \pm 0.75$ & $12.23 \pm 0.72$ & 0.811 \\
\hline
\end{tabular}

Data are presented as mean \pm S.E.M..

AFC, antral follicle count; BMI, body mass index; E2, estradiol; FSH, follicle-stimulating hormone; Gn, gonadotropin; LH, luteinizing hormone; $\mathrm{P} 4$, progesterone; PRL, prolactin; T, testosterone. 
(Time point 1, GnRH agonist day; Time point 2, Gonadotropin administration day; Time point 3, hCG administration day; Time point 4, $12 \mathrm{~h}$ after hCG administration; Time point 5, OPU day, approximately $36 \mathrm{~h}$ after hCG administration; Time point 6, $48 \mathrm{~h}$ after OPU; Time point 7, 14 days after embryo transfer). On Time points 1, 2 and 3, serum was collected at approximately 08:00 $\mathrm{h}$, before the $\mathrm{GnRH}$, gonadotropin and hCG administration. On Time point 4, serum was collected at approximately $12 \mathrm{~h}$ after the hCG injection. On Time points 5, 6 and 7, serum was collected at approximately 08:00 $\mathrm{h}$ on the defined day. After collection, serum was stored at $-80^{\circ} \mathrm{C}$ until further analysis. Pregnancy was determined by the identification of a gestational sac by abdominal ultrasound at 35 days after embryo transfer.

\section{Primary culture of human granulosa-lutein (hGL) cells}

Primary hGL cells were purified by density centrifugation from follicular aspirates collected from the same cohort of 80 patients undergoing oocyte retrieval as previously described (Fang et al. 2019). Cells were cultured in a humidified atmosphere containing $5 \% \mathrm{CO}_{2}$ and $95 \%$ air at $37^{\circ} \mathrm{C}$ in Dulbecco's Modified Eagle Medium/nutrient mixture F-12 Ham medium (DMEM/F-12; Gibco) supplemented with $10 \%$ charcoal/ dextran-treated FBS (HyClone, Shanghai, China), $100 \mathrm{U} / \mathrm{mL}$ of penicillin, and $100 \mu \mathrm{g} / \mathrm{mL}$ of streptomycin sulfate (Boster, Wuhan, China). Cells were cultured in six-well plates 5 days before performing experiments. All treatments were performed in medium containing $0.5 \%$ charcoal/dextran-treated FBS.

\section{Reverse transcription quantitative real-time PCR (RT-qPCR)}

The RT-qPCR was used to measure the levels of specific mRNA in hGL cells. Total RNA was extracted with the RNeasy Plus Mini Kit (QIAGEN) according to the manufacturer's instructions. RNA $(2 \mu \mathrm{g})$ was reverse-transcribed into first-strand cDNA with the High-Capacity cDNA Reverse Transcription Kit (Applied Biosystems). Each $20 \mu \mathrm{L}$ RT-qPCR contained 1X SYBR Green PCR Master Mix (Applied Biosystems), 60 ng of cDNA, and 250 $\mathrm{nM}$ of each specific primer. The primers used were StAR, $5^{\prime}$-AAA CTT ACG TGG CTA CTC AGC ATC-3' (sense) and 5'-GAC CTG GTT GAT GAT GCT CTT G-3' (antisense); ALK5, 5'-GTT AAG GCC AAA TAT CCC AAA CA-3' (sense) and 5'-ATA ATTTTA GCC ATT ACT CTC AAG G-3' (antisense); P450 side chain cleavage enzyme (P450scc), 5'-CAG GAG GGG TGG ACA CGA C-3' (sense) and 5'-AGG TTG CGT GCC ATC TCA TAC-3' (antisense);

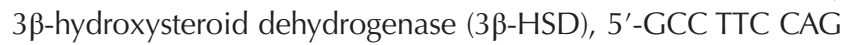
ACC AGA ATT GAG AGA-3' (sense) and 5'-TCC TTC AAG TAC AGT CAG CTT GGT-3' (antisense) and GAPDH, 5'-GAG TCA ACG GAT TTG GTC GT-3' (sense) and 5'-GAC AAG CTT CCC GTT CTC AG-3' (antisense). RT-qPCR was performed on an Applied Biosystems QuantStudio 12K Flex system equipped with 96-well optical reaction plates. The specificity of each assay was validated by melting curve analysis and by agarose gel electrophoresis of the PCR products. RT-qPCR experiments were run in triplicate, and a mean value was used to determine the mRNA levels. Water and mRNA without RT enzyme were used as negative controls. Relative quantification of mRNA levels was performed using the comparative $\mathrm{Ct}$ method with $\mathrm{GAPDH}$ as the reference gene, using the formula $2^{-\Delta \Delta \mathrm{Ct}}$.

\section{Western blotting}

The Western blotting was used to measure the levels of specific protein in hGL cells. Cells were lysed in cell lysis buffer (Cell Signaling Technology). Equal amounts of protein were separated by SDS PAGE and transferred onto PVDF membranes. After $1 \mathrm{~h}$ blocking with $5 \%$ non-fat dry milk in Tris-buffered saline (TBS), the membranes were incubated overnight at $4{ }^{\circ} \mathrm{C}$ with primary antibodies diluted in $5 \%$ nonfat milk/TBS. Following primary antibody incubation, the membranes were incubated with appropriate HRP-conjugated secondary antibodies. Immunoreactive bands were detected using an enhanced chemiluminescent substrate (Bio-Rad Laboratories, Shanghai, China) and imaged with a ChemiDoc MP Imager (Bio-Rad Laboratories).

\section{Measurement of GDF-8 and progesterone}

The ELISA was used to measure the levels of GDF-8 and progesterone in serum or follicular fluid samples. A GDF-8 ELISA kit (R\&D Systems) and a human progesterone ELISA kit (Cayman Chemical) were used in accordance with the manufacturer's protocol. All ELISA experiments were run in duplicate and a mean value was used for the determination of GDF-8 or progesterone levels.

\section{Statistical analysis}

Results are presented as the mean \pm S.E.M. of at least three independent experiments. All statistical analyses were conducted on PRISM software. For experiments involving only two groups, data were analyzed by $t$ test. Statistical significance was defined as $P<0.05$.

\section{Results \\ GDF-8 levels in serum and follicular fluid are higher in PCOS patients than in non-PCOS patients}

Given the reported contradictory results in the relationship between serum GDF-8 levels and the risk of PCOS, we first compared the serum levels of GDF-8 in patients with PCOS to the levels in patients without PCOS. Consistent with our previous study (Fang et al. 2016), ELISA results showed that GDF-8 levels in serum dynamically change during the process of $\mathrm{COH}$. Interestingly, the GDF-8 levels in serum were significantly higher on the day of gonadotropin administration (PCOS: $7.15 \pm 0.86,95 \% \mathrm{Cl}$ : 5.34-8.96 vs non-PCOS: $4.71 \pm 0.31,95 \% \mathrm{Cl}: 4.05-5.37)$ and 14 days after embryo transfer (PCOS: $5.92 \pm 0.66,95 \% \mathrm{Cl}$ : 4.52-7.32 vs non-PCOS: $4.37 \pm 0.63$, 95\% Cl: $2.97-$ 5.78 ) in PCOS patients than in non-PCOS patients. For the other examined time points, the serum GDF-8 levels did not vary significantly between PCOS patients and 

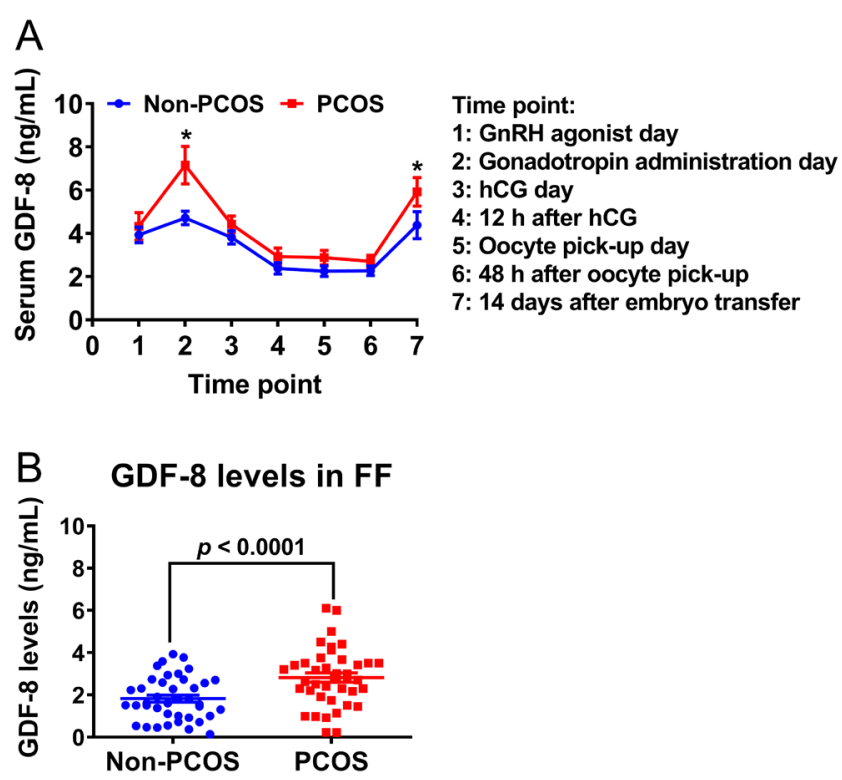

Figure 1 The expression levels of GDF-8 in serum and follicular fluid of non-PCOS patients and PCOS patients. (A and B) GDF-8 levels at different time points during the $\mathrm{COH}$ process in serum $(\mathrm{A})$ and in follicular fluid collected at the time of oocyte pick-up (B) obtained from 40 non-PCOS patients and 40 PCOS patients were measured by ELISA. The results are expressed as the mean \pm S.E.M. ${ }^{*} P<0.05$ compared between the non-PCOS group and the PCOS group at the same time point.

non-PCOS patients (Fig. 1A). Next, we examined the expression levels of GDF-8 in follicular fluid samples obtained from women undergoing oocyte retrieval during IVF treatment. As shown in Fig. 1B, GDF-8 levels were significantly higher in the follicular fluid of PCOS patients than in that of non-PCOS patients (PCOS: $2.82 \pm 0.21 \mathrm{ng} / \mathrm{mL}$ vs non-PCOS: $1.65 \pm 0.15)$.

\section{Serum and follicular GDF-8 levels are lower in pregnant PCOS patients than in non-pregnant PCOS patients}

Our previous study demonstrated that high serum and follicular fluid levels of GDF-8 were positively associated with pregnancy outcomes in non-PCOS patients (Fang et al. 2016). It remains unknown whether the same is true in PCOS patients. Therefore, we further analyzed our results by dividing the PCOS group into nonpregnant $(n=16)$ and pregnant $(n=24)$ subgroups. As shown in Fig. 2A, serum GDF-8 levels were significantly lower in the pregnant group than in the non-pregnant group during the whole process of $\mathrm{COH}$. In non-PCOS patients, consistent with our previous study (Fang et al. 2016), follicular GDF-8 levels were higher in pregnant patients than they were in non-pregnant patients. Interestingly, in contrast to the results observed in nonPCOS patients, follicular GDF-8 levels were lower in pregnant PCOS patients than they were in non-pregnant PCOS patients (Fig. 2B). These results suggest that high
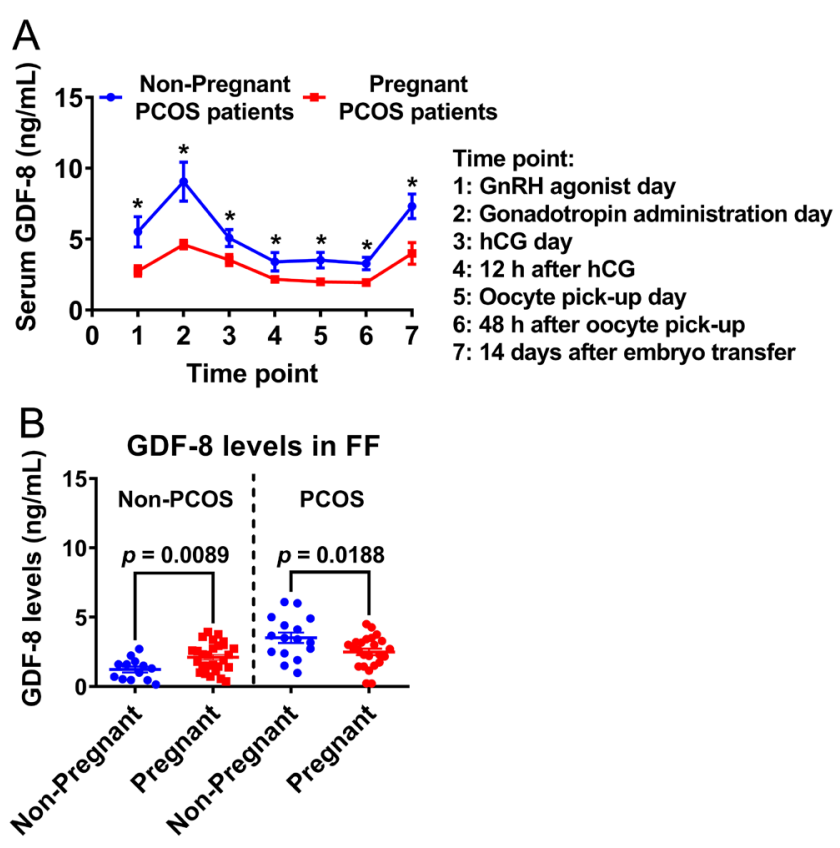

Figure 2 The expression levels of GDF-8 in serum and follicular fluid of non-pregnant and pregnant PCOS patients. The 40 non-PCOS and PCOS patients were divided into two groups according to pregnancy status (non-PCOS: 13 non-pregnant and 27 pregnant; PCOS: 16 non-pregnant and 24 pregnant). GDF-8 levels at different time points during $\mathrm{COH}$ in serum (A) and in follicular fluid (B) were measured by ELISA. The results are expressed as the mean \pm S.E.M. ${ }^{*} P<0.05$ compared between the non-pregnant group and the pregnant group at the same time point.

GDF-8 levels may negatively affect pregnancy outcomes in IVF patients with PCOS.

\section{GDF-8 inhibits StAR expression more profoundly in granulosa-lutein cells from PCOS patients than in those from non-PCOS patients}

To delineate the mechanism that mediates the effect of GDF-8 on pregnancy outcome in PCOS patients, primary cultures of human granulosa-lutein (hGL) cells from the same cohort of non-PCOS patients and PCOS patients were used as in vitro experimental models. Consistent with our previous study (Fang et al. 2015), StAR mRNA levels were significantly downregulated by exposure to GDF-8 in hGL cells from non-PCOS patients. Interestingly, the inhibitory effect of GDF-8 on StAR mRNA levels was more profoundly observed in hGL cells from PCOS patients (Fig. 3A). Similar effects of GDF-8 on StAR protein levels in non-PCOS and PCOS patients were confirmed by western blot analyses (Fig. 3B). Notably, our results showed that the basal expression levels of StAR in hGL cells from PCOS patients were lower than they were in hGL cells from non-PCOS patients (Fig. 3C). We have previously shown that the inhibitory effect of GDF-8 on StAR expression in hGL cells is mediated by the TGF- $\beta$ type I receptor, 
A

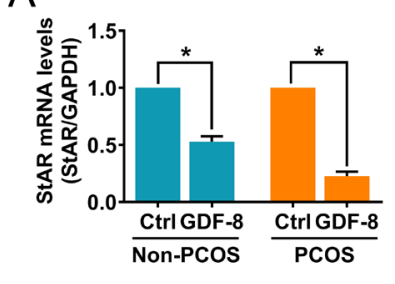

B
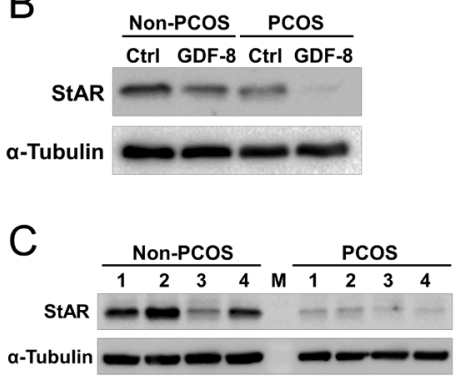
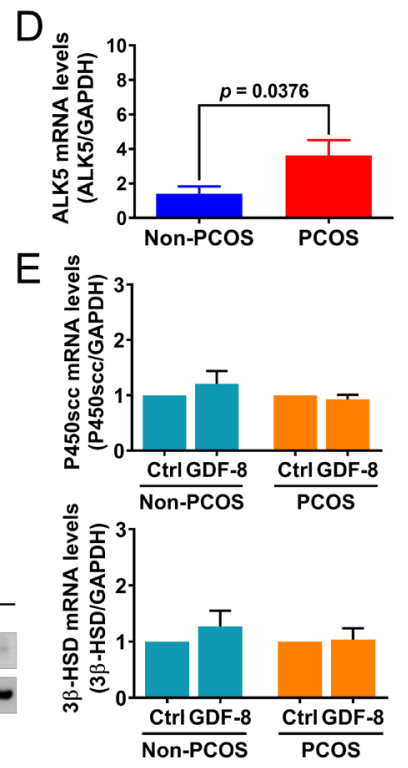

Figure 3 GDF- 8 inhibits StAR expression more profoundly in the granulosa cells from PCOS patients than in cells from non-PCOS patients. (A and B) Human granulosa-lutein ( $\mathrm{hGL}$ ) cells of non-PCOS patients and PCOS patients were treated with a vehicle control or with $30 \mathrm{ng} / \mathrm{mL}$ GDF-8 for $24 \mathrm{~h}$. StAR mRNA (A) and protein (B) levels were examined by RT-qPCR and western blot, respectively. (C) The basal protein levels of StAR were examined by western blot in 4 non-PCOS patients and 4 PCOS patients. M: protein marker. (D) The basal mRNA levels of ALK5 were examined by RT-qPCR in 9 non-PCOS patients and 9 PCOS patients. (E) Human granulosa-lutein (hGL) cells from non-PCOS patients and PCOS patients were treated with a vehicle control or with $30 \mathrm{ng} / \mathrm{mL}$ GDF-8 for $24 \mathrm{~h}$. The mRNA levels of P450scc and 3 $\beta$-HSD were examined by RT-qPCR. The results are expressed as the mean \pm S.E.M. ${ }^{*} P<0.05$ compared between Ctrl and GDF-8 treatment.

ALK5 (Fang et al. 2015). We therefore examined the expression levels of ALK5 in hGL cells from non-PCOS patients and PCOS patients. As shown in Fig. 3D, compared to non-PCOS patients, the mRNA levels of ALK5 were significantly upregulated in hGL cells from PCOS patients (Fig. 3D). The expression levels of other steroidogenesis-related enzymes, such as P450scc and $3 \beta-\mathrm{HSD}$, were not affected by exposure to GDF-8 in hGL cells from either non-PCOS patients or PCOS patients (Fig. 3E).

\section{Low serum P4 levels are observed in PCOS patients and are associated with poor pregnancy outcomes}

Given the pivotal role of StAR in the regulation of P4 production, we next examined the serum $\mathrm{P} 4$ levels during the process of $\mathrm{COH}$. As shown in Fig. 4A, serum P4 levels measured on the day of hCG administration, the oocyte pick-up (OPU) day and $48 \mathrm{~h}$ after the OPU day were lower in PCOS patients than they were in nonPCOS patients. In addition, we found that serum P4 levels were elevated from the day of hCG administration to $48 \mathrm{~h}$ after the OPU day. Interestingly, the elevation rate
A
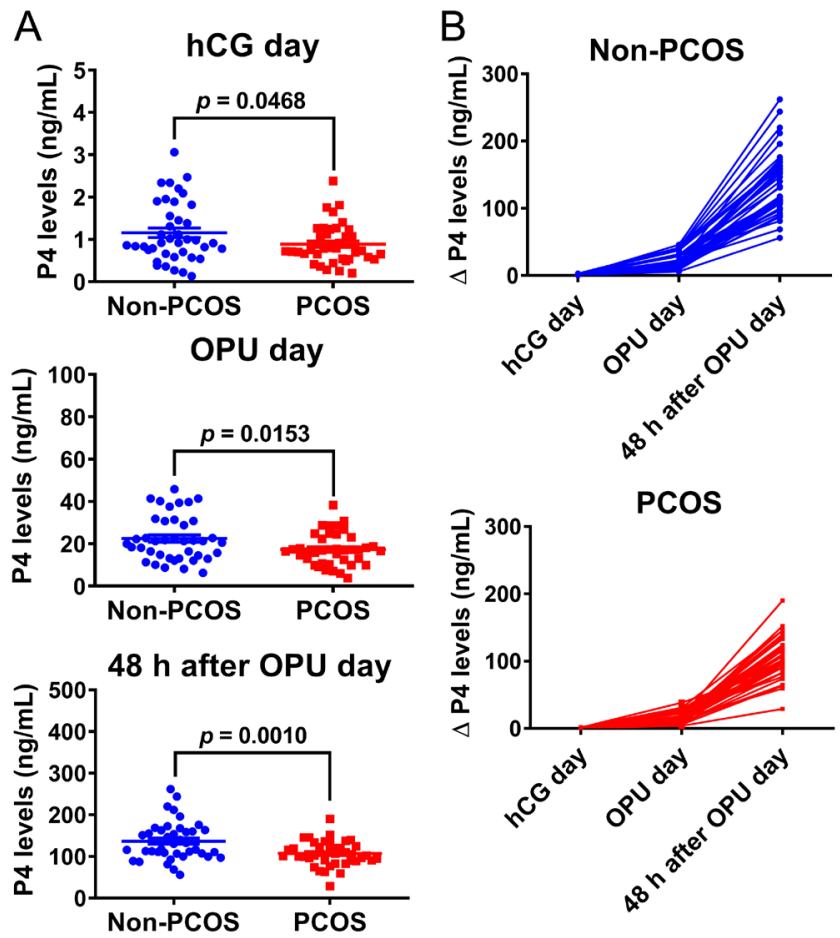

Figure 4 Serum progesterone (P4) levels in non-PCOS patients and PCOS patients during the $\mathrm{COH}$ process. (A) Serum P4 levels on the day of hCG administration (hCG day), the oocyte pick-up day (OPU day) and $48 \mathrm{~h}$ after the OPU day of 40 non-PCOS patients and 40 PCOS patients were measured by ELISA. (B) The changes in P4 levels in individual patients from the hCG day to $48 \mathrm{~h}$ after the OPU day.

was lower in PCOS patients than in non-PCOS patients. Moreover, in both non-PCOS patients and PCOS patients, serum P4 levels measured on the day of hCG administration, the OPU day and $48 \mathrm{~h}$ after the OPU day were negatively correlated with serum GDF-8 levels that measured on the same day (Fig. 5). Further analyses revealed that high serum $\mathrm{P} 4$ levels on the day of hCG administration, the OPU day and $48 \mathrm{~h}$ after the OPU day were associated with poor pregnancy outcomes in non-PCOS patients. In contrast, in PCOS patients, low serum P4 levels on the day of hCG administration, the OPU day and $48 \mathrm{~h}$ after the OPU day were associated with poor pregnancy outcomes (Fig. 6).

\section{Discussion}

PCOS, an endocrine disorder, is one of the most common causes of infertility in women. Many studies have demonstrated that aberrant hormone regulation, such as FSH deficiency, hypersecretion of LH, hyperandrogenemia and hyperinsulinemia, contributes to the pathogenesis of PCOS (Qiao \& Feng 2011). The maintenance of normal ovarian functions depends not only on endocrine regulators but also on a variety of locally produced growth factors and cytokines that exert their effects in an autocrine and/or a paracrine fashion. 


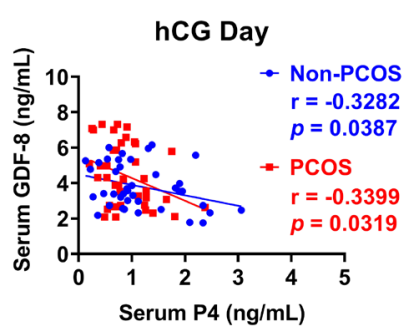

$48 \mathrm{~h}$ after OPU Day

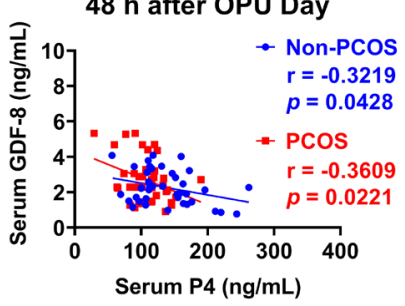

Figure 5 Serum progesterone (P4) levels are negatively correlated with serum GDF-8 levels in both non-PCOS patients and PCOS patients. Serum P4 and serum GDF-8 levels on the day of hCG administration (hCG day), the oocyte pick-up day (OPU day) and 48 $\mathrm{h}$ after the OPU day were measured by ELISA (40 non-PCOS patients and 40 PCOS patients). Pearson's correlation analyses were performed to assess their relationship.

Dysregulation of several TGF- $\beta$ superfamily members is known to contribute to the pathophysiology of PCOS (Qiao \& Feng 2011, Raja-Khan et al. 2014). Two previous studies have examined the relationship between serum GDF-8 levels and the risk of PCOS. However, contradictory results were observed (Chen et al. 2012, Arpaci 2019). This may be due to the fact that besides intra-follicular production, GDF-8 is also produced in skeletal muscle, adipose tissue and cardiomyocytes (Fan et al. 2017), which therefore contribute to the total serum concentration. In our study, we showed that during the $\mathrm{COH}$ cycle, the serum GDF-8 levels changed and were only significantly different between PCOS patients and non-PCOS patients on the day of gonadotropin administration and 14 days after embryo transfer. Taken together, these results suggest that serum levels of GDF-8 is not a good biomarker for the pathogenesis of PCOS and might explain the contradictory results observed in two previous studies (Chen et al. 2012, Arpaci 2019). Follicular fluid provides an important microenvironment for maintaining physiological functions of the ovarian follicle. In the present study, we measured GDF-8 levels in the follicular fluid and found that PCOS patients had higher follicular GDF-8 levels than non-PCOS patients did. Compared to serum, follicular fluid is not easily or routinely collected. Although it is not feasible to use follicular GDF-8 as a biomarker for PCOS, the aberrant expression of GDF-8 in follicular fluid of PCOS patients could be a potential novel target for the treatment of PCOS.

Consistent with our previous study (Fang et al. 2016), in non-PCOS patients, our results showed that GDF-8 levels
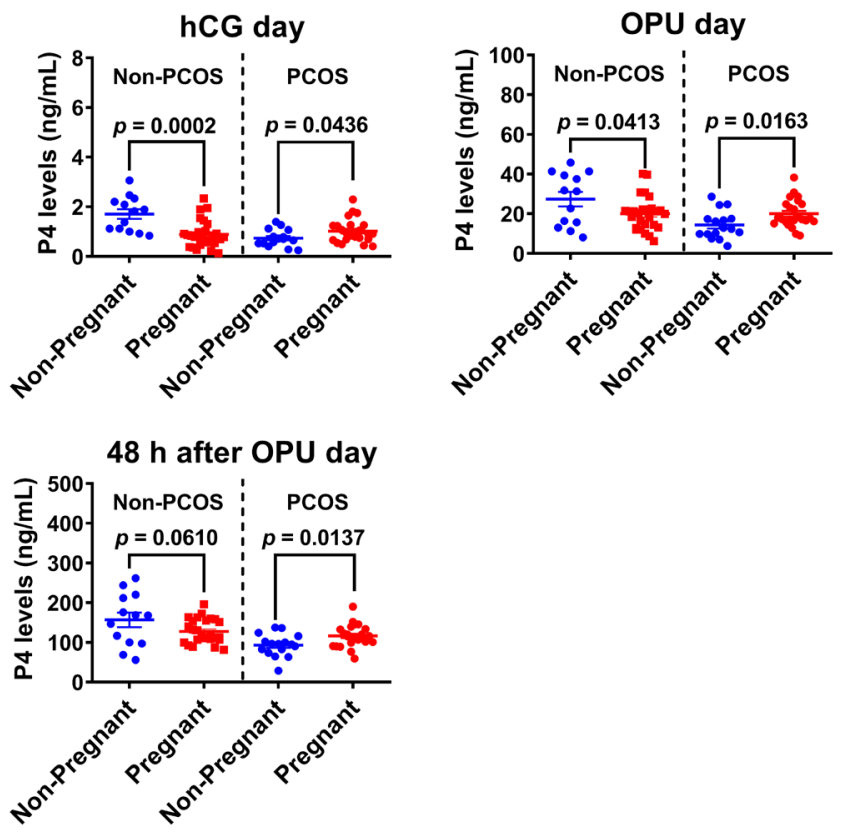

Figure 6 Serum progesterone (P4) levels in non-pregnant and pregnant PCOS patients. The 40 non-PCOS and PCOS patients were divided into two groups according to pregnancy status (non-PCOS: 13 non-pregnant and 27 pregnant; PCOS: 16 non-pregnant and 24 pregnant). The serum P4 levels on the day of hCG administration (hCG day), the oocyte pick-up day (OPU day) and $48 \mathrm{~h}$ after the OPU day were measured by ELISA.

in follicular fluid are significantly higher in pregnant patients than they are in non-pregnant patients. These data were in contrast to the results observed in PCOS patients. It is important to note that the expression levels of ALK5, the receptor mediates inhibitory effect of GDF-8 on StAR expression, were significantly higher in hGL cells from PCOS patients than in that from non-PCOS patients. In addition, the basal expression levels of StAR in hGL cells from PCOS patients were lower than they were in hGL cells from non-PCOS patients. Therefore, it is possible that high basal expression levels of GDF-8 and ALK5 could lead to an aberrant downregulation of StAR expression and $\mathrm{P} 4$ production in hGL cells of PCOS patients which might subsequently contribute to the poor pregnancy outcomes. In contrast, in non-PCOS patients, higher levels of GDF-8 might be beneficial for pregnancy by keeping a lower StAR expression and P4 levels to prevent the premature luteinization. Taken together, these findings suggest that the control of GDF-8 expression in the follicular fluid needs to be precisely regulated to ensure successful pregnancy.

It is interesting to note that $\mathrm{GnRH}$ agonist administration and embryo transfer induced a significant elevation of serum GDF-8, which was more profoundly observed in PCOS patients. However, the causes of this observation remain unknown. It is well characterized that skeletal muscle cell is the major cell type that produces GDF-8. Therefore, the levels of 
serum GDF-8 and its dynamic changes in serum may be mainly regulated by extra-follicular factors. Future studies will be needed to explore the factors and underlying mechanisms that mediate the changes of serum GDF-8 during $\mathrm{COH}$ in both non-PCOS and PCOS patients. Given the upregulation of GDF-8 expression was also observed in the follicular fluid of patients with PCOS, understanding the intra-follicular regulation machinery that contributes to the aberrant expression of GDF-8 might help design new approaches for the treatment of PCOS. Both transcriptional machinery and posttranslational machinery are reported to regulate GDF-8 gene expression (Grade et al. 2019). Several molecules and transcription factors have been shown to either stimulate or suppress the expression of GDF-8, although most of them are identified in the myogenic context of animal models (Grade et al. 2019). Nuclear factor of activated T cells (NFAT) and CRE-binding protein (CREB) are two well-known transcription factors that mediate biological functions of $\mathrm{GnRHs}$, and they are involved in IGF-1-induced GDF-8 expression in murine skeletal myoblasts (Valdes et al. 2013, Zuloaga et al. 2013, Stamatiades \& Kaiser 2018). These studies suggest that GDF-8 expression could be regulated by GnRHs. GnRHs and GnRH receptor (GnRHR) are expressed in human granulosa cells (Peng et al. 1994, Khosravi \& Leung 2003), but whether their expressions are different in the granulosa cells of PCOS patients compared to non-PCOS patients remains unknown. To date, no major defects within the GnRHR gene have been reported in association with PCOS. However, a homozygous mutation and single nucleotide polymorphisms in the GnRHR gene have been identified in PCOS patients ( $\mathrm{Li}$ et al. 2011, Caburet et al. 2017). Whether these genetic changes affect the expression of GnRHR remains to be determined.

P4 is secreted by hGL cells and is an important steroid hormone that regulates luteinization and maintains the early stages of pregnancy. Aberrant ovarian P4 secretion can lead to premature luteinization and luteal phase deficiency, which are associated with decreased implantation and pregnancy rate (Venetis et al. 2007, 2013, Practice Committee of the American Society for Reproductive Medicine 2015). Therefore, a precise modulation of ovarian $\mathrm{P} 4$ secretion is required to maintain normal reproductive functions. Previous studies have shown that $\mathrm{P} 4$ production, but not $\mathrm{E} 2$ production, is abnormal in patients with PCOS. P4 production that induced by hCG, FSH and IGF-1 is markedly reduced in the granulosa cells of PCOS patients compared to the production from the granulosa cells of non-PCOS patients (Erickson et al. 1992, Doldi et al. 1998). Similar to these findings, our results showed that, compared to non-PCOS, PCOS patients had lower serum P4 levels on the day of hCG administration, the OPU day and $48 \mathrm{~h}$ after the OPU day. In addition, after hCG administration, the elevation of serum P4 was lower in PCOS patients than in non-PCOS patients. Interestingly, a previous study demonstrated that elevated serum P4 levels on the day of hCG administration in IVF were associated with an improved pregnancy rate in PCOS patients (Doldi et al. 1999). This observation is consistent with our results that found that low serum P4 levels on the day of hCG administration, the OPU day and $48 \mathrm{~h}$ after the OPU day were associated with poor pregnancy outcomes in PCOS patients. Taken together, these results indicate that $\mathrm{P} 4$ production is dysregulated in PCOS and reveal the impact of aberrant P4 production on pregnancy outcomes in patients with PCOS.

P4 is initially synthesized from cholesterol in the mitochondria. Once free cholesterol has been transported to the mitochondria, it is further moved from the outer to the inner mitochondrial membrane by StAR protein. Therefore, StAR is recognized as the key regulator involved in the rate-limiting step of $\mathrm{P} 4$ production in granulosa cells (Clark et al. 1994, Stocco \& Clark 1996, Devoto et al. 1999). We were the first to demonstrate that GDF-8 inhibits StAR expression in hGL cells through the receptor ALK5 (Fang et al. 2015). In the present study, we showed that GDF-8 levels in serum and follicular fluid were higher in PCOS patients than in non-PCOS patients. In addition, basal levels of ALK5 were higher and basal levels of StAR were lower in PCOS patients than in nonPCOS patients. Moreover, the inhibitory effect of GDF-8 on StAR expression was more profoundly observed in hGL cells from PCOS patients than in those from nonPCOS patients. Of note, in PCOS patients, high levels of GDF-8 in serum and follicular fluid were associated with poor pregnancy outcomes. Taken together, these results could explain how high GDF-8 levels contribute to the low P4 levels and the poor pregnancy outcomes observed in PCOS patients.

In summary, the present study demonstrates that GDF-8 levels in follicular fluid are higher in IVF patients with PCOS than in those without PCOS. In PCOS patients, high follicular GDF-8 results in low P4 production by accentuating ALK5-mediated downregulation of StAR expression. Our results suggest that GDF-8 acts an important suppressor of $\mathrm{P} 4$ production by downregulating StAR expression. Aberrant expression of GDF-8 in PCOS patients results in abnormal P4 expression, which leads to poor pregnancy outcomes. This study provides an improved understanding of the role of GDF-8 in the pathogenesis of PCOS, which could lead to the development of alternative therapeutic approaches for clinical treatment of PCOS.

\section{Declaration of interest}

The authors declare that there is no conflict of interest that could be perceived as prejudicing the impartiality of the research reported. 


\section{Funding}

This work was supported by the National Key R\&D Program of China (2019YFA 0110900) and the International (Regional) Cooperation and Exchange Projects from the National Natural Science Foundation of China (81820108016) to Ying-Pu Sun. This work was also supported by the National Natural Science Foundation of China for Young Scientists (81601253) and the Key R\&D Program of Henan province (202102310062) to Lanlan Fang.

\section{Author contribution statement}

Y P S and L F contributed to the study design, analysis and interpretation of data. L F, S W, J C C and Y P S contributed to the manuscript drafting. $L F, S W, Y L, Y Y, Y L$, and $Y Y$ performed the experiments, collected clinical samples and prepared figures and table.

\section{Acknowledgments}

The authors thank all patients who donated clinical samples and data for this study. The authors also thank the staff in the Center for Reproductive Medicine at the First Affiliated Hospital of Zhengzhou University for their assistance.

\section{References}

Arpaci H 2019 Major determinants of circulating myostatin in polycystic ovary syndrome. Experimental and Therapeutic Medicine 17 1383-1389. (https://doi.org/10.3892/etm.2018.7080)

Caburet S, Fruchter RB, Legois B, Fellous M, Shalev S \& Veitia RA 2017 A homozygous mutation of GNRHR in a familial case diagnosed with polycystic ovary syndrome. European Journal of Endocrinology 176 K9K14. (https://doi.org/10.1530/EJE-16-0968)

Chang HM, Fang L, Cheng JC, Klausen C, Sun YP \& Leung PC 2015 Growth differentiation factor 8 down-regulates PENTRAXIN 3 in human granulosa cells. Molecular and Cellular Endocrinology 404 82-90. (https://doi.org/10.1016/j.mce.2015.01.036)

Chang HM, Pan HH, Cheng JC, Zhu YM \& Leung PCK 2016a Growth differentiation factor 8 suppresses cell proliferation by up-regulating CTGF expression in human granulosa cells. Molecular and Cellular Endocrinology 422 9-17. (https://doi.org/10.1016/j.mce.2015.11.009)

Chang HM, Fang L, Cheng JC, Taylor EL, Sun YP \& Leung PC $2016 b$ Effects of growth differentiation factor 8 on steroidogenesis in human granulosa-lutein cells. Fertility and Sterility 105 520-528. (https://doi. org/10.1016/j.fertnstert.2015.10.034)

Chang HM, Fang Y, Liu PP, Cheng JC, Yang X \& Leung PC 2016 c Connective tissue growth factor mediates growth differentiation factor 8 -induced increase of lysyl oxidase activity in human granulosa-lutein cells. Molecular and Cellular Endocrinology 434 186-198. (https://doi. org/10.1016/j.mce.2016.07.007)

Chen MJ, Han DS, Yang JH, Yang YS, Ho HN \& Yang WS 2012 Myostatin and its association with abdominal obesity, androgen and follistatin levels in women with polycystic ovary syndrome. Human Reproduction 27 2476-2483. (https://doi.org/10.1093/humrep/des209)

Clark BJ, Wells J, King SR \& Stocco DM 1994 The purification, cloning, and expression of a novel luteinizing hormone-induced mitochondrial protein in MA-10 mouse Leydig tumor cells. Characterization of the steroidogenic acute regulatory protein (StAR). Journal of Biological Chemistry $26928314-28322$.

Devoto L, Christenson LK, McAllister JM, Makrigiannakis A \& Strauss 3rd JF 1999 Insulin and insulin-like growth factor-I and -II modulate human granulosa-lutein cell steroidogenesis: enhancement of steroidogenic acute regulatory protein (StAR) expression. Molecular Human Reproduction 5 1003-1010. (https://doi.org/10.1093/molehr/5.11.1003)
Doldi N, Gessi A, Destefani A, Calzi F \& Ferrari A 1998 Polycystic ovary syndrome: anomalies in progesterone production. Human Reproduction 13 290-293. (https://doi.org/10.1093/humrep/13.2.290)

Doldi N, Marsiglio E, Destefani A, Gessi A, Merati G \& Ferrari A 1999 Elevated serum progesterone on the day of HCG administration in IVF is associated with a higher pregnancy rate in polycystic ovary syndrome. Human Reproduction 14 601-605. (https://doi.org/10.1093/ humrep/14.3.601)

Dumesic DA, Oberfield SE, Stener-Victorin E, Marshall JC, Laven JS \& Legro RS 2015 Scientific statement on the diagnostic criteria, epidemiology, pathophysiology, and molecular genetics of polycystic ovary syndrome. Endocrine Reviews 36 487-525. (https://doi. org/10.1210/er.2015-1018)

Erickson GF, Magoffin DA, Garzo VG, Cheung AP \& Chang RJ 1992 Granulosa cells of polycystic ovaries: are they normal or abnormal? Human Reproduction 7 293-299. (https://doi.org/10.1093/ oxfordjournals.humrep.a137638)

Fan X, Gaur U, Sun L, Yang D \& Yang M 2017 The growth differentiation factor 11 (GDF11) and myostatin (MSTN) in tissue specific aging. Mechanisms of Ageing and Development 164 108-112. (https://doi. org/10.1016/j.mad.2017.04.009)

Fang L, Chang HM, Cheng JC, Yu Y, Leung PC \& Sun YP 2015 Growth differentiation factor-8 decreases StAR expression through ALK5mediated Smad3 and ERK1/2 signaling pathways in luteinized human granulosa cells. Endocrinology 156 4684-4694. (https://doi.org/10.1210/ en.2015-1461)

Fang L, Yu Y, Zhang R, He J \& Sun YP 2016 Serum GDF-8 levels change dynamically during controlled ovarian hyperstimulation in patients undergoing IVF/ICSI-ET. Scientific Reports 6 28036. (https://doi. org/10.1038/srep28036)

Fang L, Yu Y, Li Y, Wang S, Zhang R, Guo Y, Li Y, Yan Y \& Sun YP 2019 Human chorionic gonadotropin-induced amphiregulin stimulates aromatase expression in human granulosa-lutein cells: a mechanism for estradiol production in the luteal phase. Human Reproduction 34 2018-2026. (https://doi.org/10.1093/humrep/dez171)

Grade CVC, Mantovani CS \& Alvares LE 2019 Myostatin gene promoter: structure, conservation and importance as a target for muscle modulation. Journal of Animal Science and Biotechnology 1032. (https://doi.org/10.1186/s40104-019-0338-5)

Khosravi S \& Leung PC 2003 Differential regulation of gonadotropinreleasing hormone $(\mathrm{GnRH}) \mathrm{I}$ and $\mathrm{GnRHII}$ messenger ribonucleic acid by gonadal steroids in human granulosa luteal cells. Journal of Clinical Endocrinology and Metabolism 88 663-672. (https://doi.org/10.1210/ jc.2002-020866)

Knight PG \& Glister C 2006 TGF-beta superfamily members and ovarian follicle development. Reproduction 132 191-206. (https://doi. org/10.1530/rep.1.01074)

Li Q, Yang G, Wang Y, Zhang X, Sang Q, Wang H, Zhao X, Xing Q, He L \& Wang L 2011 Common genetic variation in the 3 '-untranslated region of gonadotropin-releasing hormone receptor regulates gene expression in cella and is associated with thyroid function, insulin secretion as well as insulin sensitivity in polycystic ovary syndrome patients. Human Genetics 129 553-561. (https://doi.org/10.1007/s00439-011-0954-4)

Lin TT, Chang HM, Hu XL, Leung PCK \& Zhu YM 2018 Follicular localization of growth differentiation factor 8 and its receptors in normal and polycystic ovary syndrome ovaries. Biology of Reproduction 98 683-694. (https://doi.org/10.1093/biolre/ioy029)

McCartney CR \& Marshall JC 2016 CLINICAL PRACTICE. Polycystic ovary syndrome. New England Journal of Medicine 375 54-64. (https://doi. org/10.1056/NEJMcp1514916)

McPherron AC \& Lee SJ 1997 Double muscling in cattle due to mutations in the myostatin gene. PNAS 94 12457-12461. (https://doi.org/10.1073/ pnas.94.23.12457)

McPherron AC, Lawler AM \& Lee SJ 1997 Regulation of skeletal muscle mass in mice by a new TGF-beta superfamily member. Nature 387 83-90. (https://doi.org/10.1038/387083a0)

Peng C, Fan NC, Ligier M, Vaananen J \& Leung PC 1994 Expression and regulation of gonadotropin-releasing hormone $(\mathrm{GnRH})$ and $\mathrm{GnRH}$ receptor messenger ribonucleic acids in human granulosaluteal cells. Endocrinology 135 1740-1746. (https://doi.org/10.1210/ endo.135.5.7956897)

Practice Committee of the American Society for Reproductive Medicine 2015 Current clinical irrelevance of luteal phase deficiency: a committee 
opinion. Fertility and Sterility 103 e27-e32. (https://doi.org/10.1016/j. fertnstert.2014.12.128)

Qiao J \& Feng HL 2011 Extra- and intra-ovarian factors in polycystic ovary syndrome: impact on oocyte maturation and embryo developmental competence. Human Reproduction Update 17 17-33. (https://doi. org/10.1093/humupd/dmq032)

Raja-Khan N, Urbanek M, Rodgers RJ \& Legro RS 2014 The role of TGFbeta in polycystic ovary syndrome. Reproductive Sciences 21 20-31. (https://doi.org/10.1177/1933719113485294)

Rotterdam ESHRE/ASRM-sponsored PCOS consensus workshop group 2004 Revised 2003 consensus on diagnostic criteria and long-term health risks related to polycystic ovary syndrome (PCOS). Human Reproduction 19 41-47. (https://doi.org/10.1093/humrep/deh098)

Stamatiades GA \& Kaiser UB 2018 Gonadotropin regulation by pulsatile GnRH: signaling and gene expression. Molecular and Cellular Endocrinology $\mathbf{4 6 3}$ 131-141. (https://doi.org/10.1016/j. mce.2017.10.015)

Stocco DM \& Clark BJ 1996 Regulation of the acute production of steroids in steroidogenic cells. Endocrine Reviews 17 221-244. (https://doi. org/10.1210/edrv-17-3-221)

Valdes JA, Flores S, Fuentes EN, Osorio-Fuentealba C, Jaimovich E \& Molina A 2013 IGF-1 induces IP3 -dependent calcium signal involved in the regulation of myostatin gene expression mediated by NFAT during myoblast differentiation. Journal of Cellular Physiology 228 1452-1463. (https://doi.org/10.1002/jcp.24298)

Venetis CA, Kolibianakis EM, Papanikolaou E, Bontis J, Devroey P \& Tarlatzis BC 2007 Is progesterone elevation on the day of human chorionic gonadotrophin administration associated with the probability of pregnancy in in vitro fertilization? A systematic review and metaanalysis. Human Reproduction Update 13 343-355. (https://doi. org/10.1093/humupd/dmm007)

Venetis CA, Kolibianakis EM, Bosdou JK \& Tarlatzis BC 2013 Progesterone elevation and probability of pregnancy after IVF: a systematic review and meta-analysis of over 60000 cycles. Human Reproduction Update 19 433-457. (https://doi.org/10.1093/humupd/dmt014)

Witchel SF, Oberfield SE \& Peña AS 2019 Polycystic ovary syndrome: pathophysiology, presentation, and treatment with emphasis on adolescent girls. Journal of the Endocrine Society 3 1545-1573. (https:// doi.org/10.1210/js.2019-00078)

Zuloaga R, Fuentes EN, Molina A \& Valdes JA 2013 The cAMP response element binding protein (CREB) is activated by insulin-like growth factor-1 (IGF-1) and regulates myostatin gene expression in skeletal myoblast. Biochemical and Biophysical Research Communications 440 258-264. (https://doi.org/10.1016/j.bbrc.2013.09.067)

Received 8 February 2020

First decision 11 March 2020

Revised manuscript received 30 March 2020

Accepted 9 April 2020 